ARTICLE

\title{
Genetically encoded photocrosslinkers locate the high-affinity binding site of antidepressant drugs in the human serotonin transporter
}

Hafsteinn Rannversson 1,2, Jacob Andersen', Lena Sørensen¹, Benny Bang-Andersen 1,3, Minyoung Park², Thomas Huber ${ }^{2}$, Thomas P. Sakmar ${ }^{2,4}$ \& Kristian Strømgaard ${ }^{1}$

Despite the well-established role of the human serotonin transporter (hSERT) in the treatment of depression, the molecular details of antidepressant drug binding are still not fully understood. Here we utilize amber codon suppression in a membrane-bound transporter protein to encode photocrosslinking unnatural amino acids (UAAs) into 75 different positions in hSERT. UAAs are incorporated with high specificity, and functionally active transporters have similar transport properties and pharmacological profiles compared with wild-type transporters. We employ ultraviolet-induced crosslinking with $p$-azido-L-phenylalanine (azF) at selected positions in hSERT to map the binding site of imipramine, a prototypical tricyclic antidepressant, and vortioxetine, a novel multimodal antidepressant. We find that the two antidepressants crosslink with azF incorporated at different positions within the central substrate-binding site of hSERT, while no crosslinking is observed at the vestibular-binding site. Taken together, our data provide direct evidence for defining the high-affinity antidepressant binding site in hSERT.

\footnotetext{
${ }^{1}$ Department of Drug Design and Pharmacology, Center for Biopharmaceuticals, University of Copenhagen, Jagtvej 162, DK-2100 Copenhagen, Denmark. ${ }^{2}$ Laboratory of Chemical Biology \& Signal Transduction, The Rockefeller University, 1230 York Avenue, New York, New York 10065, USA. ${ }^{3}$ Lundbeck Research Denmark, H. Lundbeck A/S, Ottiliavej 9, DK-2500 Valby, Denmark. ${ }^{4}$ Department of Neurobiology, Care Sciences and Society, Center for Alzheimer Research, Karolinska Institutet, Huddinge 141 57, Sweden. Correspondence and requests for materials should be addressed to K.S. (email: kristian.stromgaard@sund.ku.dk).
} 
C ommunication between the cells of the nervous system is mediated by chemical neurotransmitters that are released from vesicles in the presynaptic neuron, diffuse across the synapse and bind to their target receptor proteins on the postsynaptic neuron. Synaptic signal transmission is regulated by the reuptake of released neurotransmitter from the synapse into the neurons by integral membrane-bound neurotransmitter transporters ${ }^{1}$. The serotonin transporter (SERT) is a member of the solute carrier 6 family of transporters, which also includes the transporters for the neurotransmitters norepinephrine, dopamine, glycine and $\gamma$-aminobutyric acid ${ }^{2}$. SERT controls the magnitude and duration of serotonergic neurotransmission by facilitating sodium-, potassium- and chloride-dependent reuptake of released serotonin (5-hydroxytryptamine; 5-HT) against its concentration gradient ${ }^{2}$. Pharmacological modulation of SERT transport activity influences a variety of neurophysiological processes, and inhibitors of SERT have been extensively used in the treatment of psychiatric diseases, such as depression and anxiety, with $>30$ drugs in current clinical use ${ }^{3}$.

$\mathrm{X}$-ray crystal structures of bacterial and invertebrate homologues of SERT, including the bacterial amino acid transporters MhsT and LeuT ${ }^{4-6}$, and the Drosophila melanogaster dopamine transporter $(\mathrm{dDAT})^{7-10}$, have proven to be excellent structural templates for their mammalian counterparts. These structures have suggested inhibitor binding at two distinct sites: the central substrate-binding site (designated the S1 site) and a vestibular site (designated the S2 site), which is located on the extracellular side of the S1 site (Fig. 1a). While mutational studies of SERT ${ }^{11-16}$, in combination with crystal structures of $\mathrm{dDAT}^{7-10}$, have suggested that antidepressants bind within the S1 site, selective serotonin reuptake inhibitors (SSRIs) and tricyclic antidepressants (TCAs) bind in the S2 site of LeuT ${ }^{17-20}$. The S2 site was first described as a secondary substrate-binding site in LeuT $^{21}$, but has also recently been suggested to harbour an allosteric inhibitor-binding site in human transporters ${ }^{22}$. The precise role of the S2 site in inhibitor binding is therefore under debate, and studies that can unequivocally address this issue are much needed.

Site-specific incorporation of light-sensitive unnatural amino acids (UAAs) into proteins is a powerful approach to investigate molecular mechanisms as well as biological function with high spatial and temporal resolution. We therefore decided to employ amber codon suppression technology to introduce photocrosslinking UAAs into SERT. The methodology relies on the recognition of a nonsense codon, such as the low-abundance amber (UAG) stop codon, in the gene of interest using an orthogonal transfer RNA (tRNA) that is aminoacylated with a UAA by an aminoacyl tRNA synthetase (aaRS) ${ }^{23-26}$. Specifically, the photocrosslinking amino acids $p$-azido-L-phenylalanine (azF) and $p$-benzoyl-L-phenylalanine (BzF; Fig. 1b), which crosslink on ultraviolet radiation, have been used to gain detailed insight into protein-protein interactions ${ }^{27-30}$. Importantly, azF and BzF have also been incorporated into integral membrane protein drug targets, such as $G$ protein-coupled receptors ${ }^{31-38}$ and ionotropic receptors ${ }^{39-41}$, to understand receptor function and to map ligand-binding sites. However, the technology has so far not been employed in studies of neurotransmitter transporters.

We demonstrate that the photocrosslinking UAAs azF and BzF can be introduced into human SERT (hSERT) with high efficiency and specificity. We further find that imipramine, a prototypical TCA, and vortioxetine, a novel multimodal antidepressant, irreversibly crosslink to azF located at different positions in the S1-binding site of hSERT, while no crosslinking is observed when azF is inserted into the S2-binding site. Our results prove the feasibility of introducing genetically encoded photoreactive UAAs into a neurotransmitter transporter and also provide direct evidence for the location of the high-affinity inhibitor-binding site in hSERT.

\section{Results}

hSERT is amenable to the incorporation of UAAs. To explore the feasibility of incorporating the photocrosslinking amino acids azF and BzF (Fig. 1b) into hSERT in a comprehensive and systematic manner, we co-transfected HEK293T cells with plasmids carrying the genes of hSERT containing an amber codon at a desired position along with an orthogonal suppressor tRNA/aaRS pair for azF or BzF. The cells were cultured in media containing either azF or BzF, and subsequently the translational efficiency and fidelity were assessed in a functional $\left[{ }^{3} \mathrm{H}\right] 5-\mathrm{HT}$ uptake assay. We probed 75 positions in hSERT, representing different side-chain structures and spatial locations in the protein (Fig. 1d), where azF or BzF was introduced through amber codon suppression mutagenesis, resulting in 150 hSERT mutants.

In the majority of the tested positions, azF or BzF could be genetically encoded into hSERT and led to a functionally active transporter protein (uptake activity of $>5 \%$ compared with wildtype (WT) hSERT). Specifically, of the 75 positions examined, the functional activity was retained in $55(73 \%)$ of the azF mutants and in $47(63 \%)$ of the BzF mutants (Fig. 1d, Supplementary Results; Supplementary Fig. 1; Supplementary Table 1). Our comprehensive data set allowed us to evaluate putative correlations between the nature of the native amino acid, as well as spatial location, and efficiency of incorporation of azF and BzF (Supplementary Table 2). Generally, mutations of native aromatic residues to either azF or BzF were better tolerated compared with mutations of non-aromatic residues ( $96 \%$ versus $65 \%$ for azF; $78 \%$ versus $56 \%$ for $\mathrm{BzF}$ ). The incorporation of $\mathrm{BzF}$ at the transmembrane segments was tolerated at $20 \%$ of the positions tested, while $44 \%$ of the transmembrane mutants retained functionality for azF incorporation. The loop and termini regions were highly amenable to both azF and BzF incorporation with only a single terminal BzF mutant not being functional (Supplementary Table 2). Furthermore, the specificity and fidelity of azF incorporation were determined at five different positions of the transporter (Y95, W103, Y175, F341 and K490; Fig. 1d), by measuring the activity in cells where one of the transfection components (tRNA, aaRS or azF) was omitted (Fig. 1c). Negligible functional activity $(<10 \%)$ was observed when suppressor tRNA or aaRS was absent from the transfections. In the absence of azF, generally a markedly reduced activity was observed. However, for K490 in particular, we did observe some read-through in the absence of azF, which is due to imperfect fidelity for azF by the engineered aaRS/tRNA pair ${ }^{35}$.

Introduction of azF into the $\mathrm{S} 1$ and $\mathrm{S} 2$ sites in hSERT After establishing the amber codon suppression technique for incorporation of photocrosslinking UAAs into hSERT, we wanted to explore if UAAs could be introduced in regions of hSERT relevant for mapping of drug-binding sites. The initial screen of 75 residues was used to identify functionally active mutants surrounding the putative inhibitor-binding sites (S1 and S2). Due to a better incorporation tolerance and generally higher activities of azF mutants compared with BzF mutants, we chose to focus on azF mutants. We selected five mutants surrounding the S1-binding site (Y95azF, I168azF, Y175azF, F335azF and F341azF) and five mutants surrounding the S2-binding site (W103azF, Y107azF, W182azF, Y487azF and K490azF), based on spatial orientation and distance from the proposed binding sites in a homology model of hSERT ${ }^{42}$ (Fig. 2a). Initially, we analysed the expression of the 10 azF mutants by immunoblotting. 
a

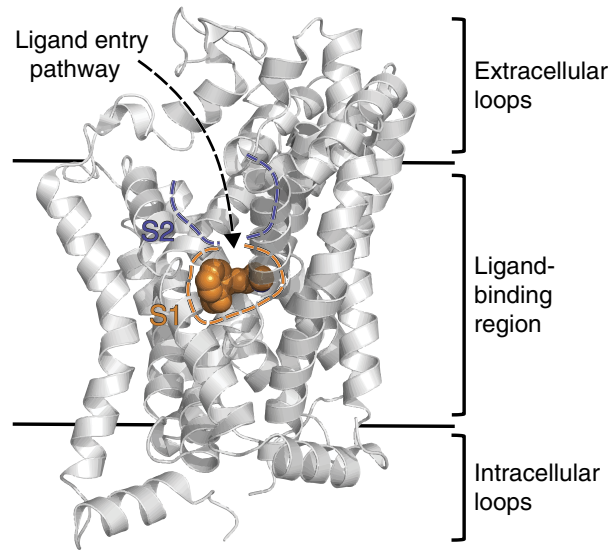

b<smiles>NC(Cc1ccc(N=[N+]=[OH+])cc1)C(=O)O</smiles>

C

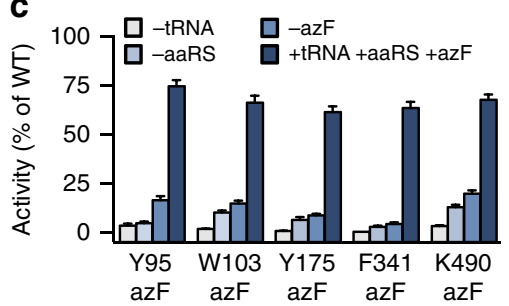

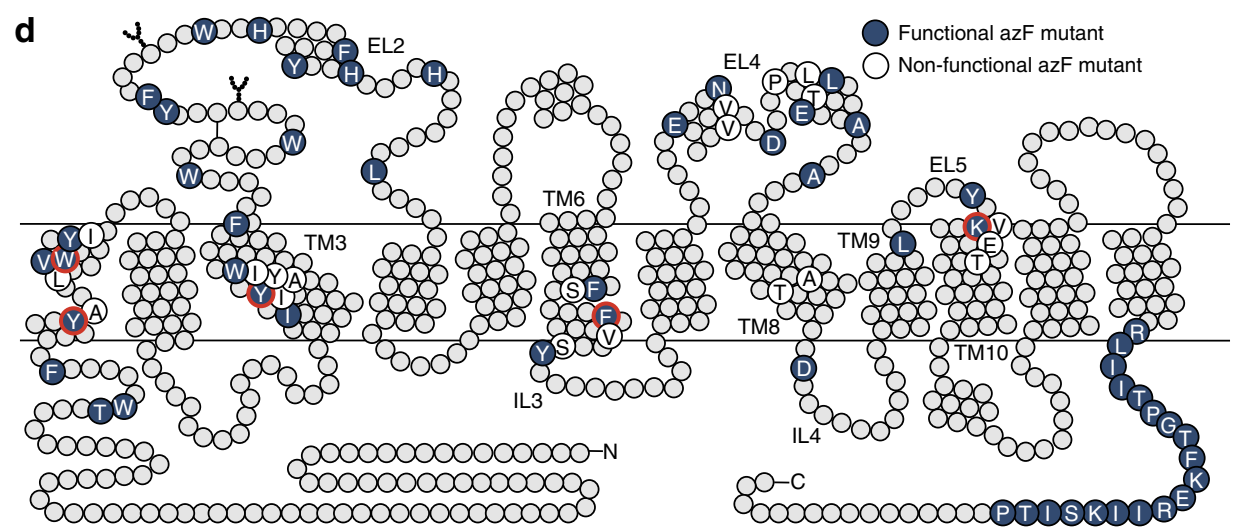

Figure 1 | Introduction of photocrosslinking UAAs into hSERT. (a) X-ray crystal structure of dDAT with nortriptyline bound (PDB ID 4M48). The location of the S1- and S2-binding sites are illustrated with orange and purple dashed lines, respectively. TM10 and TM11 have been removed for clarity. (b) Chemical structures of azF and BzF. (c) Specificity of azF incorporation. The $\left[{ }^{3} \mathrm{H}\right] 5-\mathrm{HT}$ uptake activity of five azF mutants (Y95azF, W103azF, Y175azF, F341azF and K490azF) where one of the transfection components (suppressor tRNA, aaRS or azF) was omitted. Data are represented as mean \pm s.e.m. from three independent experiments each performed in triplicate. (d) Two-dimensional schematic representation of hSERT showing the location of the 75 positions that were mutated to azF or BzF. Blue circles indicate positions where azF introduction resulted in a functionally active transporter (defined as $>5 \%$ functional activity relative to WT hSERT activity), while white circles indicate positions where introduction of azF abolished activity (see Supplementary Table 1 and Supplementary Fig. 1 for transport activity of BzF mutants). Circles highlighted with a red line designate the positions examined in c. TM, transmembrane.

The immunoblot analyses were performed on HEK293T cells expressing azF mutants of hSERT, using Rho1D4 monoclonal antibody $(\mathrm{mAb})$ against a C-terminal 1D4 epitope fused to hSERT to ensure that only full-length protein would be detected. A clear band was detected for WT hSERT and each of the $10 \mathrm{azF}$ mutants at $75-80 \mathrm{kDa}$, corresponding to mature glycosylated hSERT ${ }^{43}$. Consistent with the lower activity of the $10 \mathrm{azF}$ mutants compared with WT hSERT (Supplementary Table 1), the intensities of the bands corresponding to the azF mutants were weaker than the band from WT hSERT in all cases, indicating a lower expression level of the mutants. Importantly, no band corresponding to full-length hSERT was detected in any of the samples from cells grown in the absence of azF (Fig. 2c).

To investigate whether the incorporation of azF affected the transport kinetics, cells expressing azF mutants of hSERT were incubated with increasing concentrations of the substrate (5-HT) and the maximum rate of transport $\left(V_{\max }\right)$ and the substrate concentration needed to achieve half maximum rate of transport $\left(K_{\mathrm{m}}\right)$ were determined. Introduction of azF into the S1 or S2 site led to decreased $V_{\max }$ values, whereas the $K_{\mathrm{m}}$ values of the mutants were in the same range as WT hSERT, with the exception of F335azF, F341azF and K490azF, where the $K_{\mathrm{m}}$ values decreased by 6- to 13-fold (Fig. 2b; Supplementary Table 3). Thus, despite a lower transport activity caused by lower cell surface expression (Fig. 2c), the $10 \mathrm{azF}$ mutants retained sufficient uptake activity for robust and reliable measurements.

Mapping of drug-binding sites using amber codon suppression. Next, the potential of the amber codon suppression technique for mapping of the drug-binding sites in hSERT was investigated. Specifically, we examined whether antidepressant drugs could undergo ultraviolet-induced crosslinking to any of the $10 \mathrm{~S} 1 / \mathrm{S} 2 \mathrm{azF}$ mutants of hSERT, and thereby provide unambiguous evidence that the drug binds in close proximity $(<3 \AA)^{31}$ to the given azF side chain. We chose two pharmacologically and structurally different antidepressant drugs for these studies: the classical TCA drug imipramine and vortioxetine, a newly marketed multimodal antidepressant drug, which in addition to potent inhibition of hSERT (Fig. 3a,b) also binds to and modulates the activity of different serotonergic receptors ${ }^{44,45}$. To determine the binding affinity of the two drugs for WT hSERT, we performed saturation binding assays using $\left[{ }^{3} \mathrm{H}\right]$ labelled versions of imipramine and vortioxetine, and found that WT hSERT binds vortioxetine and imipramine with dissociation constants $\left(K_{\mathrm{d}}\right)$ of $6.3 \pm 0.7$ and $7.2 \pm 0.8 \mathrm{nM}$, respectively (Fig. 3a,b; Supplementary Fig. 2).

To examine whether the $10 \mathrm{~S} 1 / \mathrm{S} 2$ azF mutants affected the potency of the two drugs, the inhibition constants $\left(K_{\mathrm{i}}\right)$ of vortioxetine and imipramine for WT hSERT and the $10 \mathrm{azF}$ 
a

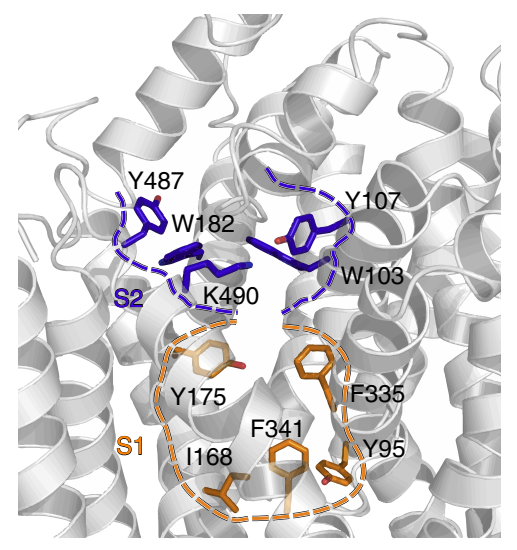

b

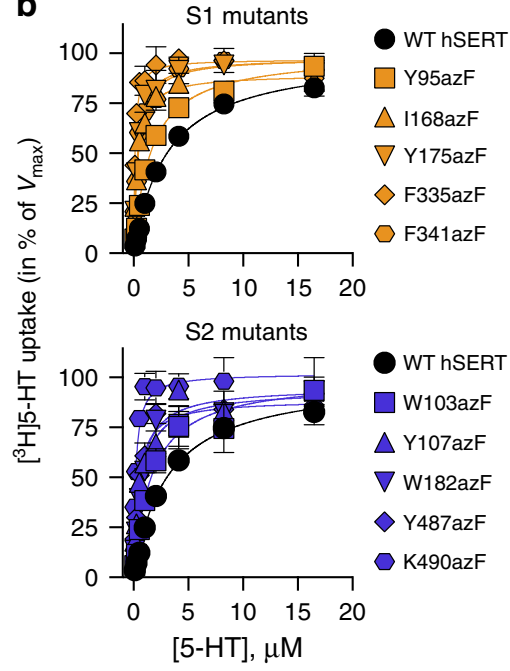

C

\begin{tabular}{|c|c|c|c|c|c|c|c|c|c|c|}
\hline \multirow[b]{2}{*}{$\frac{k \mathrm{Da}}{80-1}$} & \multicolumn{5}{|c|}{ S1 mutants } & \multicolumn{5}{|c|}{ S2 mutants } \\
\hline & $\begin{array}{l}\text { Y95 } \\
\text { azF }\end{array}$ & $\begin{array}{l}1168 \\
\text { azF }\end{array}$ & $\begin{array}{l}\text { Y175 } \\
\text { azF }\end{array}$ & $\begin{array}{c}\text { F335 } \\
\text { azF }\end{array}$ & $\begin{array}{c}\mathrm{F} 341 \\
\text { azF }\end{array}$ & $\begin{array}{c}\text { W103 } \\
\text { azF }\end{array}$ & $\begin{array}{c}\text { Y107 } \\
\text { azF }\end{array}$ & $\begin{array}{c}\text { W182 } \\
\text { azF }\end{array}$ & $\begin{array}{c}\text { Y487 } \\
\text { azF }\end{array}$ & $\begin{array}{c}\mathrm{K} 490 \\
\mathrm{azF}\end{array}$ \\
\hline & & +9 & & & $=$ & & 8 & 6 & ! & in \\
\hline
\end{tabular}

Figure 2 | Characterization of hSERT containing photocrosslinking UAAs around the S1- and S2-binding sites. (a) Homology model of hSERT showing the residues in the S1- and S2-binding sites that were mutated to azF. Residues located within the S1 and S2 sites are shown in orange and purple, respectively. (b) $\left[{ }^{3} \mathrm{H}\right] 5-\mathrm{HT}$ uptake saturation curves (normalized to the maximal rate of transport, $V_{\max }$ ) in HEK293T cells expressing WT hSERT and the five S1 azF mutants (orange symbols) or the five S2 azF mutants (purple symbols), illustrating the effects of the azF mutations on the uptake kinetics of the transporter (see Supplementary Table 3 for $K_{m}$ and $V_{\max }$ values). Data points are represented as mean \pm s.e.m. from a representative experiment with triplicate determinations. (c) Immunoblot analysis of lysates from HEK293T cells expressing WT hSERT or azF mutants, using a mAb against a C-terminal 1D4 epitope fused to hSERT. Using an epitope at the $\mathrm{C}$ terminus of the transporter, only full-length protein is detected in the immunoblot analysis. Cells were grown either in the presence or absence of azF. For the azF mutants, no full-length protein was detected in the absence of azF, illustrating that translation stopped when the amber stop codon was reached.

mutants were determined in a $\left[{ }^{3} \mathrm{H}\right] 5$-HT uptake inhibition assay (Fig. 3c,d). Generally, the S2-binding site mutations had modest effects on the potency of imipramine and vortioxetine, except for the Y107azF mutant, which was 5-fold and 12-fold more sensitive to vortioxetine and imipramine, respectively (Fig. 3c,d; Supplementary Table 3). More noticeable changes were seen for the S1-binding site mutants. The most pronounced effects were seen for I168azF, which was significantly more sensitive to both vortioxetine and imipramine (10-fold and 33-fold, respectively), whereas Y95azF decreased the potency of both compounds (6-fold and 21-fold, respectively; Fig. 3c,d; Supplementary Table 3). Our mutational data indicated that the inhibitors bind to the central substrate site in hSERT, and is thus consistent with the fact that imipramine bind competitively with $5-\mathrm{HT}^{46}$. Furthermore, we found that vortioxetine does not change the $V_{\max }$ value but significantly increases the $K_{\mathrm{m}}$ value of 5-HT transport (Supplementary Fig. 3), strongly suggesting that vortioxetine also binds competitively with 5-HT in the central substrate site of hSERT.

Since all $10 \mathrm{~S} 1 / \mathrm{S} 2$ mutants bound both the inhibitors, we investigated their ability to form covalent crosslinks to $\left[{ }^{3} \mathrm{H}\right]$ labelled drugs. In these experiments, WT hSERT and the $10 \mathrm{azF}$ mutants were transfected into the same batch of HEK293T cells and the 10 mutants and WT hSERT were assayed in parallel with each drug. Specifically, HEK293T cells expressing either WT or azF mutants of hSERT were incubated with $100 \mathrm{nM}$ of either $\left[{ }^{3} \mathrm{H}\right]$ vortioxetine or $\left[{ }^{3} \mathrm{H}\right]$ imipramine at $4{ }^{\circ} \mathrm{C}$ for $2 \mathrm{~h}$ to reach binding equilibrium (Fig. 4a). After incubation, cells expressing a given azF mutant were divided into two batches, one of which was exposed to ultraviolet light to induce the chemical crosslinking reaction, while the other was used as a control to assess the level of non-ultraviolet-induced crosslink. After cell lysis and immunopurification of the transporters using Rho1D4 $\mathrm{mAb}$, the samples were separated by SDS-polyacrylamide gel (SDS-PAGE) electrophoresis and protein transferred onto a polyvinylidene fluoride (PVDF) membrane for immunoblotting. Each sample lane of the PVDF membrane was subsequently divided into three segments containing protein with the molecular weights of $20-50,50-100$ or $>100 \mathrm{kDa}$ (Fig. 4a). Consistent with the initial immunoblot analyses (Fig. 2c), the bands corresponding to hSERT were located in the $50-100-\mathrm{kDa}$ segments (Supplementary Fig. 6). Finally, each membrane piece was placed in a scintillation vial containing scintillation fluid to quantify the radioactivity of each sample (Fig. 4a), where a radioactivity signal indicated irreversible chemical crosslink between the azF hSERT mutant and the $\left[{ }^{3} \mathrm{H}\right]$ labelled drug.

Importantly, no radioactivity signal was observed in WT hSERT after incubation of either $\left.{ }^{3} \mathrm{H}\right]$ vortioxetine or $\left[{ }^{3} \mathrm{H}\right]$ imipramine, showing that the ultraviolet-induced crosslinking did not occur when azF was not genetically encoded (Fig. 4b,c; Supplementary Fig. 4). Examination of the radioactivity patterns for the two inhibitors at the $10 \mathrm{azF}$ mutants revealed significant insight into the specificity of crosslinking. For $\left[{ }^{3} \mathrm{H}\right]$ imipramine, there was no difference between the radioactivity signals from the ultraviolet-exposed samples and the control samples at any of the S2-binding site mutants, showing that $\left[{ }^{3} \mathrm{H}\right]$ imipramine did not crosslink to any of these mutants. In contrast, the radioactivity signal was significantly higher in the ultraviolet-induced sample of the S1 mutant Y95azF compared with the control sample, showing that $\left[{ }^{3} \mathrm{H}\right]$ imipramine was 

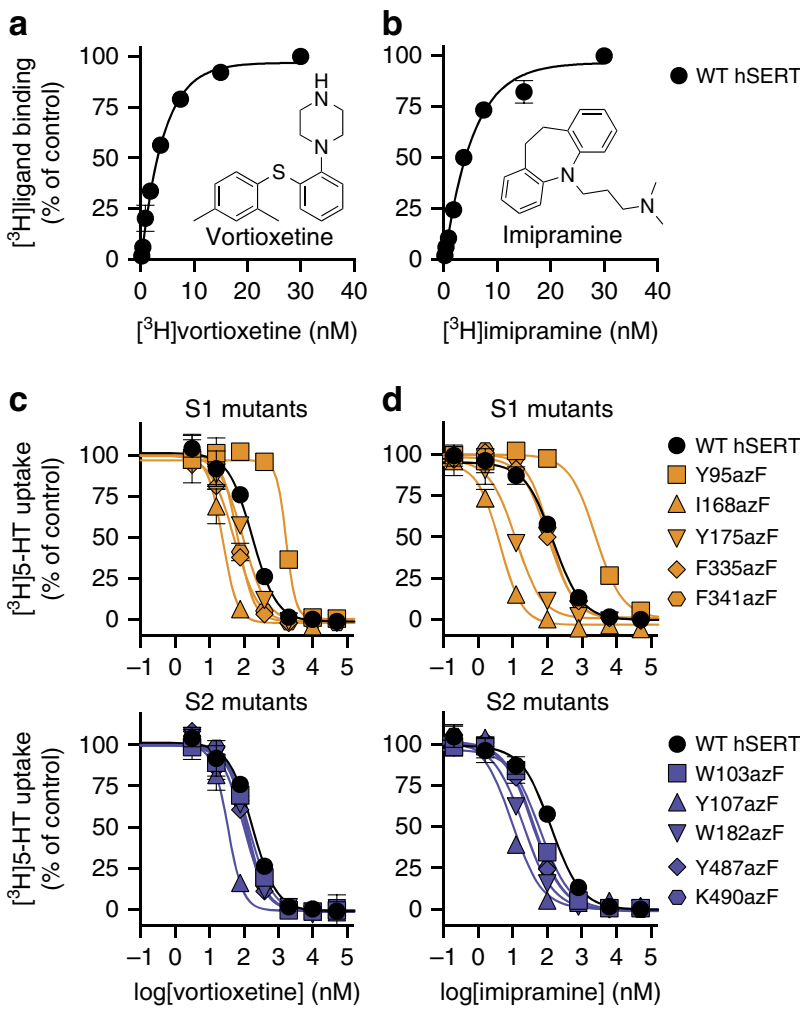

Figure 3 | Binding of vortioxetine and imipramine to hSERT.

(a) Saturation binding curve for $\left[{ }^{3} \mathrm{H}\right]$ vortioxetine binding to HEK293T membranes expressing WT hSERT. Vortioxetine binds to WT hSERT with a $K_{d}$ of $6.3 \pm 0.7 \mathrm{nM}$ (mean \pm s.e.m. from 11 independent experiments each performed in triplicate). (b) Saturation binding curve for $\left[{ }^{3} \mathrm{H}\right]$ imipramine binding to HEK293T membranes expressing WT hSERT. Imipramine binds to WT hSERT with a $K_{\mathrm{d}}$ of $7.2 \pm 0.8 \mathrm{nM}$ (mean \pm s.e.m. from six independent experiments each performed in triplicate). (c,d) Inhibition of $\left[{ }^{3} \mathrm{H}\right] 5-\mathrm{HT}$ uptake by vortioxetine (c) or imipramine (d) in HEK293T cells expressing WT hSERT, one of the five S1-binding site mutants (orange symbols) or one of the five S2-binding site mutants (purple symbols), illustrating the effect of the mutations on the potency of vortioxetine and imipramine. $K_{i}$ values for vortioxetine and imipramine on WT hSERT and the 10 azF mutants are included in Supplementary Table 3. Each data point represents mean \pm s.e.m. from a representative experiment carried out in triplicate. A C-terminal 1D4 epitope was fused to WT hSERT and 10 azF mutants. Importantly, this epitope did not affect the binding affinity of vortioxetine or imipramine (Supplementary Fig. 2).

covalently attached to the Y95azF mutant upon ultraviolet radiation (Fig. 4b). The signal was detected in the $50-100 \mathrm{kDa}$ segment of the membrane, in agreement with the size of mature glycosylated SERT, while no radioactivity was detected in the $20-50$ or $>100 \mathrm{kDa}$ membrane segments (Supplementary Fig. 4). Similarly, no radioactivity signal was detected in any of the control samples that were not exposed to ultraviolet light, demonstrating that crosslinking of $\left[{ }^{3} \mathrm{H}\right]$ imipramine was dependent on ultraviolet exposure (Fig. 4b).

For $\left[{ }^{3} \mathrm{H}\right]$ vortioxetine, the radioactivity signals for ultravioletinduced and control samples were also indistinguishable for the five S2 mutants (Fig. 4c). Interestingly, a different pattern was observed with the S1 mutants for vortioxetine compared with imipramine. A significant ultraviolet-dependent radioactivity signal was detected at F341azF when incubated with $\left[{ }^{3} \mathrm{H}\right]$ vortioxetine, while no signal was detected at Y95azF, which elicited a signal for imipramine (Fig. 4c). The signal at F341azF was exclusively detected in the $50-100 \mathrm{kDa}$ membrane segment
(Supplementary Fig. 4). Furthermore, a full displacement of the radioactivity signal at $\mathrm{Y} 95 \mathrm{azF}$ and $\mathrm{F} 341 \mathrm{azF}$ for imipramine and vortioxetine, respectively, was observed when $100 \mu \mathrm{M}$ of unlabelled inhibitor was added before ultraviolet exposure, further demonstrating the specificity of the signal (Fig. 4b,c).

From these results, we conclude that the radioactivity signals detected at Y95azF and F341azF for imipramine and vortioxetine, respectively, were a result of direct ultraviolet-induced crosslink between the $\left[{ }^{3} \mathrm{H}\right]$ labelled drugs and azF inserted into hSERT, thereby providing direct evidence for inhibitor binding in the S1-binding site of hSERT.

\section{Discussion}

Considering the role of hSERT as a principal drug target in the treatment of affective disorders, obtaining a better understanding of drug binding is of great clinical importance. Intensive efforts to understand the molecular basis of antidepressant binding to hSERT have mainly relied on site-directed mutagenesis and cysteine accessibility studies. However, these studies do not provide direct experimental information about inhibitor binding, and it has been difficult to assign specific inhibitor-binding functionalities unambiguously based on these mutational studies due to potential indirect and long-range allosteric effects on protein structure. Photoaffinity analogues of tropane-based inhibitors, such as cocaine, and SSRIs, including escitalopram, have been developed to define drug-protein interactions at the molecular level in DAT and SERT, respectively ${ }^{47-50}$. However, although photoaffinity ligands can provide a defined anchor point for ligand orientation within a binding pocket, addition of the relatively large photoreactive functional group to a drug likely affects its binding mode. X-ray crystal structures of dDAT co-crystallized with antidepressants suggest that the high-affinity inhibitor-binding site in hSERT overlaps the central S1 site $\mathrm{s}^{7,8,10}$. In contrast, studies on hSERT have suggested that inhibitor binding is not limited to the S1 site 22 , which is also supported by X-ray crystal structures of LeuT complexed with TCAs and SSRIs in the S2 region ${ }^{17-19}$. Since no X-ray crystal structures of hSERT in complex with antidepressants have yet been obtained, many important uncertainties remain regarding inhibitor binding at hSERT.

In the present study, we demonstrate the use of amber codon suppression in a membrane transporter protein and report direct evidence for the location of the high-affinity antidepressantbinding site in hSERT. Specifically, we show that two structurally and pharmacologically different antidepressant drugs, imipramine and vortioxetine, can be covalently attached to azF when this photocrosslinking UAA is inserted into the central S1 site in hSERT, thereby directly showing that the binding site for vortioxetine and imipramine overlaps the central substratebinding site. In contrast, no ultraviolet-induced photocrosslinking to imipramine or vortioxetine was observed when azF was inserted into the vestibular S2 site of hSERT (Fig. 4b,c). Noteworthy, since we were aiming at mapping the high-affinity binding site for vortioxetine and imipramine, we employed a concentration of $100 \mathrm{nM}$ of the drugs in the photocrosslinking experiments. Under these conditions, a low-affinity binding site may not be occupied, and the lack of covalent photocrosslinking signal in S2, cannot rule out the presence of a secondary, allosteric inhibitor-binding site in this vestibular site.

The S1 site in hSERT is a narrow and constrained area that is located in the core of the transporter protein (Fig. 1a). Previously established binding models for imipramine ${ }^{51}$ and vortioxetine ${ }^{42}$ in hSERT suggest that both the drugs primarily interact through hydrophobic interactions to S1 residues in addition to formation of a salt bridge between an aspartate residue (Asp98) and a 
a
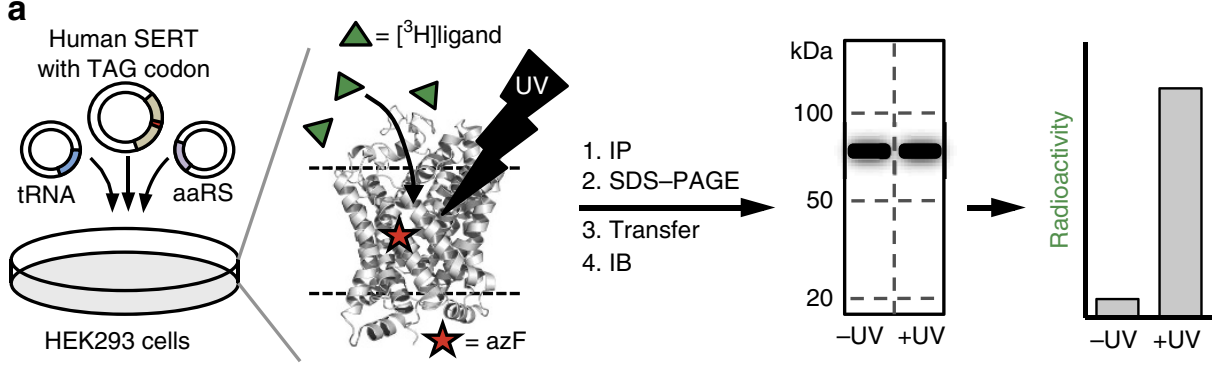

b
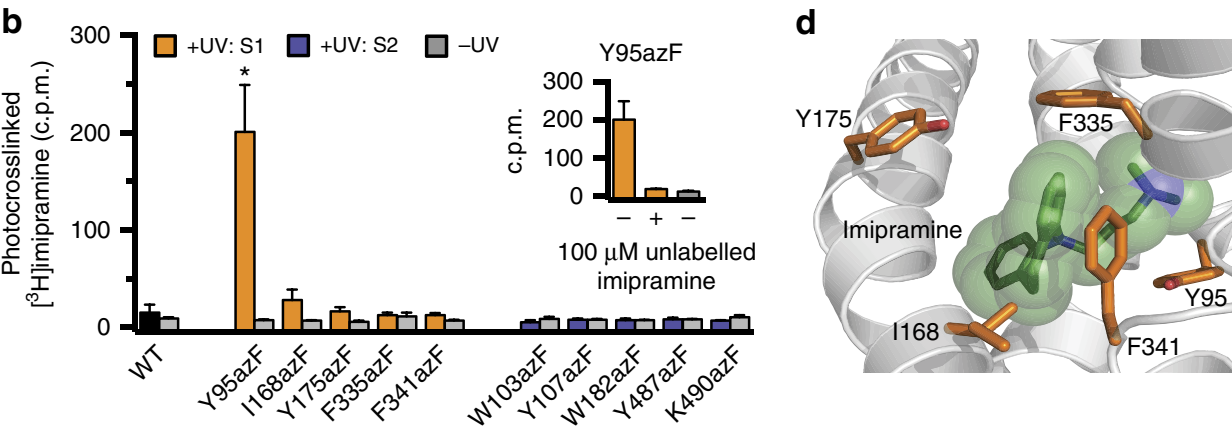

C

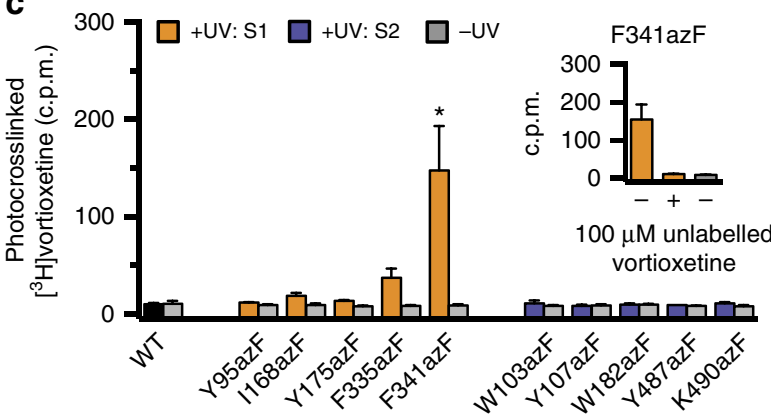

e

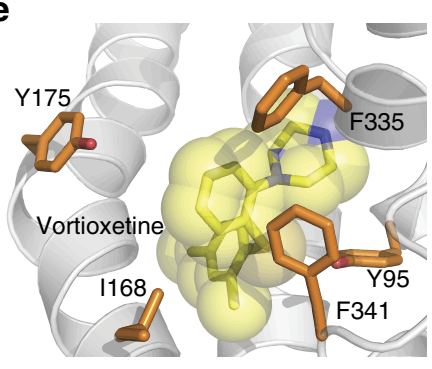

Figure 4 | Crosslinking studies and binding site determination of vortioxetine and imipramine. (a) Schematic illustration outlining the photocrosslinking experiments. Briefly, HEK293T cells expressing WT or azF mutants of hSERT were incubated with $100 \mathrm{nM}$ of $\left[{ }^{3} \mathrm{H}\right]$ vortioxetine or $\left[{ }^{3} \mathrm{H}\right]$ imipramine and exposed to ultraviolet (UV) light. Cells were lysed, the transporter immunopurified (IP) and run on an SDS-PAGE gel, before transferring to PVDF membrane for immunoblotting (IB). Each lane of the membrane was cut into three segments (20-50, 50-100 and $>100 \mathrm{kDa}$, respectively) and the radioactivity of each segment was quantified. As control, non-UV-exposed samples were in all cases assayed in parallel. (b,c) Results from the photocrosslinking experiments between hSERT azF mutants, and $\left[{ }^{3} \mathrm{H}\right]$ imipramine $(\mathbf{b})$ and $\left[{ }^{3} \mathrm{H}\right]$ vortioxetine (c). The bars represent the radioactivity signal expressed as counts per minute (c.p.m.) detected in PVDF membrane segments corresponding to 50-100 kDa that contain hSERT. For the 20-50 and $>100 \mathrm{kDa}$ segments, the radioactivity signal was in all cases indistinguishable between UV-exposed and control samples (Supplementary Fig. 4). Asterisks $\left.{ }^{*}\right)$ denotes significant difference in crosslinking signal compared with non-UV-exposed samples $(P<0.05$; two-way analysis of variance with Sidak multiple comparisons test). Inserts show that crosslink of $\left[{ }^{3} \mathrm{H}\right]$ imipramine to $\mathrm{Y} 95 \mathrm{azF}(\mathbf{b})$ and $\left[{ }^{3} \mathrm{H}\right]$ vortioxetine to $\mathrm{F} 341 \mathrm{azF}$ (c) was fully outcompeted in the presence of $100 \mu \mathrm{M}$ unlabelled inhibitor added before UV exposure. Data are represented as mean \pm s.e.m. from three to seven independent experiments. $(\mathbf{d}, \mathbf{e})$ Docking models of imipramine ${ }^{51}$ (d) and vortioxetine ${ }^{42}(\mathbf{e})$ binding in the S1 site in hSERT. Imipramine is shown in green (d), vortioxetine is shown in yellow (e) and the five S1 residues that were mutated to azF are shown as orange sticks.

positively charged nitrogen atom in the drugs. Furthermore, Tyr95 has been suggested as a key interaction site for both imipramine and vortioxetine $\mathrm{e}^{16,42,51,52}$, and we indeed found that the Y95azF mutant decreased the potency of the two drugs (Fig. 3c,d). We also found that inserting azF into position 168 (I168azF) in the bottom of the S1 site increased the potency of both inhibitors (Fig. 3c,d). Similarly, mutation of Ile168 has previously been shown to increase the potency of vortioxetine and other inhibitors, such as fluoxetine $e^{42,53}$. Ile168 is assumed to be located close to the aromatic moieties of vortioxetine and imipramine (Fig. 4d,e), and the increased inhibitor binding could be mediated by additional hydrophobic interactions. Interestingly, we found that azF mutants in the S2 site generally induced an increased potency of both imipramine and vortioxetine (Fig. 3c,d; Supplementary Table 3). Most prominent was the effect of Y107azF, which induced 5- and 12-fold increase in potency of vortioxetine and imipramine, respectively. Since the inhibitors have to permeate through the S2 site to reach the central S1 site (Fig. 1a), the increased potency could be induced by altering the kinetics of ligand binding (that is, by inducing a faster association to or a slower dissociation from the S1 site) or the S2 mutations could alter the conformational equilibrium of the transporter, and thereby allosterically affect ligand binding in S1. It is noteworthy that some of the azF mutants induce 6- to 13fold decreased $K_{\mathrm{m}}$ value as found for F335azF, F341azF and K490azF (Fig. 2b; Supplementary Table 3). This is in line with previous studies showing that other mutations in the same positions such as F335W, F341Y and K490F induce 6- to 16-fold decreased $K_{\mathrm{m}}$ values in hSERT $^{13,14}$, and thus substantiates that these three residues hold an important role for substrate 
recognition and/or are important determinants for the conformational equilibrium. Specifically, F335 forms a conserved hydrophobic lid together with Y176 close to the top of the S1 site, and movement of the aromatic side chain of F335 has been proposed to be an important trigger for the shift between outward-open and inward-open conformations ${ }^{10}$. The decreased $K_{\mathrm{m}}$ value in $\mathrm{F} 335 \mathrm{azF}$ could thus be induced by altering the conformational equilibrium of hSERT.

Interestingly, all of the five positions in which azF was introduced into the S1-binding pocket (Y95, I168, Y175, F335 and F341) are located in close proximity of the proposed binding sites of imipramine ${ }^{51}$ and vortioxetine ${ }^{42}$, and thereby represent potential candidates for undergoing ultraviolet-induced photocrosslinking to the drug molecules (Fig. 4d,e; Supplementary Fig. 5). Importantly, primary conclusions can only be made in cases where a positive crosslinking signal is observed, and a positive crosslink is only possible if there is a close physical interaction between the engineered transporter and the ligand in the presence of ultraviolet light. We found that vortioxetine and imipramine were only covalently attached to a single, but different, azF mutant within the S1 site (Y95azF for imipramine; F341azF for vortioxetine). For imipramine, it is notable that Y95azF displayed the highest degree of photocrosslinking even when this azF mutant decreased the potency of the drug (Fig. 3d). The decreased potency could result from the added bulk of the azide group relative to the phenolic hydroxyl, but the significant photocrosslinking to Y95azF unequivocally show that imipramine bind in close proximity of Y95 within the S1 site as proposed previously ${ }^{16,52}$. Upon ultraviolet illumination, azF forms the highly reactive nitrone that can undergo nucleophile addition resulting in covalent crosslinking ${ }^{54}$. The amino groups of vortioxetine and imipramine can serve as nucleophiles in crosslink reactions with azF, and these nucleophilic moieties thus represent obvious crosslinking attachment sites on the drugs. Docking models of vortioxetine and imipramine in hSERT (Fig. 4d,e; Supplementary Fig. 5) show that Y95 is located closer $(\sim 1 \AA)$ to the dimethylamino moiety of imipramine, compared with the piperazine ring of vortioxetine. In contrast, F341 is located $>1 \AA$ closer to the piperazine ring of vortioxetine compared with the dimethylamino group of imipramine. Hence, the proposed docking models of vortioxetine and imipramine in hSERT are consistent with the selective photocrosslinking to $\mathrm{Y} 95 \mathrm{azF}$ and $\mathrm{F} 341 \mathrm{azF}$ that we found for imipramine and vortioxetine, respectively.

In summary, we have shown direct evidence of antidepressant binding at hSERT using a targeted photocrosslinking strategy with genetically encoded UAAs. The amber codon suppression technology applied to neurotransmitter transporters has allowed site-specific crosslinking of two unmodified antidepressant drugs in the S1-binding site of hSERT at distinct juxtaposed UAA side chains. We believe that this general method will be important in future studies that will provide additional functional and mechanistic insights into this important class of membrane proteins, including molecular level insights into transporter structure and function.

\footnotetext{
Methods

Materials. DMEM, fetal bovine serum, trypsin and penicillin-streptomycin was purchased from Life Technologies. DNA restriction enzymes and T4 DNA ligase enzyme were from New England Biolabs. Cell culture dishes were from Sarstedt AG \& Co, and white 96-well plates were from Nunc. $\left[{ }^{3} \mathrm{H}\right] 5-\mathrm{HT}$ $\left(27-28 \mathrm{Ci} \mathrm{mmol}^{-1}\right),\left[{ }^{3} \mathrm{H}\right]$ imipramine $\left(41 \mathrm{Ci} \mathrm{mmol}^{-1}\right)$, MicroScint-0 and -20 scintillation fluid and 96-well glass fibre filter plates were obtained from PerkinElmer Life Sciences. $\left[{ }^{3} \mathrm{H}\right]$ vortioxetine $\left(69 \mathrm{Ci} \mathrm{mmol}^{-1}\right)$ was purchased from Ubichem Pharma Services (Hungary). azF and BzF were from Chem-Impex International, Inc. Rho1D4 mAb was obtained from The National Cell Culture Center.
}

Molecular biology. Plasmids containing the suppressor tRNA, azF aaRS or BzF aaRS ${ }^{35,36}$ and the plasmid pcDNA3.1 hSERT ${ }^{13}$ were used for transfections. Unless otherwise noted, all experiments were conducted on hSERT constructs C-terminally fused to the 1D4 epitope. The 1D4 epitope, TETSQVAPA, was added to hSERT by replacing the coding sequence for WT hSERT with a PCR-generated insert containing the coding sequence for hSERT carrying the C-terminal 1D4 epitope. PCR primers were purchased from MWG Eurofins with restriction sites for XhoI and XbaI for the $5^{\prime}$ and $3^{\prime}$ primers, respectively. Generation of amber codon (TAG) mutations in SERT-1D4 was performed by site-directed mutagenesis using the QuickChange mutagenesis kit (Stratagene). 1D4 tag insertion and amber codon mutations were verified by DNA sequencing of the entire coding domain sequence (GATC Biotech).

Cell culturing and expression of SERT azF and BzF mutants. HEK293T cells (American Type Culture Collection, Manassas, VA) were cultured in DMEM media with $10 \%$ fetal bovine serum, $100 \mathrm{U} \mathrm{ml}^{-1}$ penicillin and $100 \mu \mathrm{g} \mathrm{ml}^{-1}$ streptomycin at $37^{\circ} \mathrm{C}$ in a humidified $5 \% \mathrm{CO}_{2}$ environment ${ }^{16}$. Cells were transfected at $60-70 \%$ confluency using TransIT LT-1 (Mirus) or Lipofectamine 2000 (Life Technologies) transfection reagent, following the protocol supplied by the manufacturer. The plasmid DNA ratio hSERT/suppressor tRNA/aaRS was $1 / 1 / 0.1$ by mass. For transfections with lipofectamine in a $10-\mathrm{cm}$ plate, $8.25 \mu \mathrm{g}$ of hSERT amber codon mutant DNA was used with $52 \mu \mathrm{l}$ of lipofectamine 2000. For transfections with TransIT LT-1 in a 96-well plate, $4.8 \mu \mathrm{g}$ of hSERT amber codon mutant DNA was used with $30 \mu \mathrm{l}$ of TransIT LT-1. For transfection in other types of plates, amounts were scaled according to surface area. Transfected cells were grown in media supplemented with $0.5 \mathrm{mM}$ of either azF or BzF

Uptake assays. Uptake assays were performed $40-48 \mathrm{~h}$ post transfection in 96-well plates. Cells were washed twice with PBSCM buffer (in $\mathrm{mM}: \mathrm{NaCl}, 137$; $\left.\mathrm{KCl}, 2.7 ; \mathrm{Na}_{2} \mathrm{HPO}_{4}, 4.3 ; \mathrm{KH}_{2} \mathrm{PO}_{4}, 1.4 ; \mathrm{CaCl}_{2}, 0.1 ; \mathrm{MgCl}_{2}, 0.5\right)$ on an ELx50 automated microplate strip washer (BioTek Instruments) and left with phosphatebuffered saline containing $\mathrm{CaCl}_{2}$ and $\mathrm{MgCl}_{2}$ (PBSCM) for 20 min before uptake experiments. For uptake activity studies, cells were incubated with $50 \mu \mathrm{l}$ PBSCM containing $50 \mathrm{nM}\left[{ }^{3} \mathrm{H}\right] 5-\mathrm{HT}$. For the determination of $K_{\mathrm{m}}$ and $V_{\max }$ values, cells were incubated with $50 \mu \mathrm{l}$ of PBSCM containing increasing concentrations of $\left[{ }^{3} \mathrm{H}\right] 5-\mathrm{HT}$ (diluted 1:10 or 1:20 with cold 5-HT). In inhibition studies (half-maximal inhibitory concentration $\left(\mathrm{IC}_{50}\right)$ determinations), cells were preincubated in $40 \mu \mathrm{l}$ of PBSCM with increasing concentrations of inhibitor for $30 \mathrm{~min}$ before the addition of $20 \mu$ of PBSCM containing $\left[{ }^{3} \mathrm{H}\right] 5$-HT giving a final substrate concentration of $200 \mathrm{nM}$. In all experiments, uptake was allowed to proceed for $5 \mathrm{~min}$ at $20^{\circ} \mathrm{C}$ and was terminated by washing three times with PBSCM (ELx50 automated microplate strip washer). The amount of accumulated radiolabelled substrate was determined by solubilizing cells in scintillation fluid (MicroScint-20), followed by counting of plates in a Packard TopCounter. All control experiments on WT hSERT were carried out in the background of the

co-transfected orthogonal azF or BzF tRNA/aaRS pair, with cells incubated in the presence of azF or BzF. Non-specific uptake was determined in parallel as uptake in non-transfected HEK293T cells. Uptake assays were carried out in triplicate and repeated at least three times unless otherwise noted.

Cell membrane preparation and radioligand-binding assay. Membranes were prepared from HEK293T cells transiently expressing hSERT growing on $15-\mathrm{cm}$ tissue culture plates. At $40-48 \mathrm{~h}$ post transfection, cells were suspended in PBS and centrifuged at low speed $(700 \mathrm{~g})$ for $5 \mathrm{~min}$. The cell pellet was resuspended in cold $\mathrm{H}_{2} \mathrm{O}$ and frozen at $-20^{\circ} \mathrm{C}$ for $1 \mathrm{~h}$. The suspension was then thawed on ice and subjected to $10-15$ passages through a $21-$ Ga needle to disrupt cells. The homogenate was centrifuged at $18,000 \mathrm{~g}$ at $4{ }^{\circ} \mathrm{C}$ for $30 \mathrm{~min}$, the supernatant fraction was aspirated and the pellet was resuspended in PBSCM. Protein concentration was determined by the BCA method using Pierce bicinchoninic acid (BCA) Protein Assay (ThermoFischer). Membranes were used directly for binding assays or stored at $-80^{\circ} \mathrm{C}$ until use. For saturation binding studies, increasing concentrations of $\left[{ }^{3} \mathrm{H}\right]$ imipramine or $\left[{ }^{3} \mathrm{H}\right]$ vortioxetine were incubated with 5 or $15 \mu \mathrm{g}$ total membrane protein per sample in a total volume of $150 \mu \mathrm{l}$ PBSCM. Binding proceeded for $2 \mathrm{~h}$ on ice with gentle rocking. Subsequently, membranes were transferred to 96-well glass fibre filter plates preincubated with $0.1 \%$ polyethyleneimine $(30 \mu \mathrm{l}$ per well) using a Packard Bell cell harvester and washed four times with water. Nonspecific binding was determined in parallel with membranes from non-transfected HEK293T cells. Filter plates were dried and soaked in scintillation fluid (MicroScint-0), followed by counting in a Packard Topcounter. Saturation binding assays were carried out in triplicate and repeated at least three times.

Immunoaffinity purification. HEK293T cells growing in 15-cm culture dishes and transiently expressing 1D4-tagged hSERT mutants were suspended in PBS 40-48 h post-transfection, pelleted by centrifugation $(700 \mathrm{~g}, 5 \mathrm{~min})$ and solubilized $\left(2 \mathrm{~h}, 4^{\circ} \mathrm{C}\right)$ in solubilization buffer $(50 \mathrm{mM}$ Tris- $\mathrm{HCl}, \mathrm{pH} 7.5,145 \mathrm{mM} \mathrm{NaCl}$, $5 \mathrm{mM}$ EDTA and $1 \%$ Triton X-100 or IGEPAL CA-630, supplemented with protease inhibitors (cOmplete, Roche)). After centrifugation (18,000g, $30 \mathrm{~min})$, the supernatant fraction was incubated overnight at $4{ }^{\circ} \mathrm{C}$ with Dynabeads Protein G (Life Technologies) bound to Rho1D4 mAb or with sepharose 2B beads 
conjugated to Rho1D4 mAb. Beads were washed five times with solubilization buffer and Dynabeads Protein G bound samples were eluted for $10 \mathrm{~min}$ at $70^{\circ} \mathrm{C}$ with LDS loading buffer (Life Technologies), while sepharose bead samples were eluted twice for $20 \mathrm{~min}$ at $60^{\circ} \mathrm{C}$ with LDS loading buffer.

Gel electrophoresis and immunoblot analysis. Protein samples were subjected to SDS-PAGE on a $4-12 \%$ Bis-Tris gel (Life Technologies) and transferred to a PVDF membrane. Detection was performed using the SNAP i.d. Protein Detection System (Millipore). PBS-T (0.1\% Tween 20 in PBS) was used as washing buffer and $0.2 \%$ non-fat dried milk in PBS-T was used as blocking reagent. Blots were incubated 10 min with Rho1D4 mAb (1:1,000 dilution of a $1 \mathrm{mg} \mathrm{ml}^{-1}$ stock), followed by 10 -min incubation with a horseradish peroxidase-conjugated anti-mouse $\mathrm{Ab}$ (1:20,000 dilution of a $1 \mathrm{mg} \mathrm{ml}^{-1}$ stock) (Promega). Blots were developed using enhanced chemoluminescence detection reagents (GE Healthcare) and visualized using a DNR MicroChemi System (DNR Bio-Imaging Systems, Ltd). For photocrosslinking studies, the immunoblot analysis was performed using TBS-T ( $0.1 \%$ Tween 20 in $20 \mathrm{mM}$ TBS) as washing buffer and $5 \%$ non-fat dried milk in TBS-T as blocking buffer. Blots were incubated with primary Rho1D4 mAb (1:3000 in $5 \%$ blocking buffer) overnight at $4{ }^{\circ} \mathrm{C}$. After five washes with TBS-T, the PVDF membrane was incubated $1 \mathrm{~h}$ at room temperature with secondary anti-mouse $\mathrm{Ab}$ conjugated with horseradish peroxidase (1:20,000 in 5\% blocking buffer). The blot was developed using Supersignal West Pico Chemiluminescent Substrate (Thermo Scientific) before being exposed to HyBlot CL auto-radiography film (Denville Scientific Inc.). See Supplementary Fig. 6 for representative western blot of azF mutants of hSERT.

Photocrosslinking of $\left[{ }^{3} \mathbf{H}\right]$ vortioxetine and $\left[{ }^{3} \mathbf{H}\right]$ imipramine. At $40-48 \mathrm{~h}$ post transfection, HEK293T cells growing in $10-\mathrm{cm}$ culture dishes and transiently expressing 1D4 epitope-tagged WT or azF hSERT mutants were pelleted by centrifugation $(700 \mathrm{~g}, 5 \mathrm{~min}$ ) and resuspended in Hank's Buffered Salt Solution ( $\mathrm{pH} 7.5$ ) containing $20 \mathrm{mM}$ HEPES, $0.2 \%$ bovine serum albumin and $100 \mathrm{nM}$ of either $\left[{ }^{3} \mathrm{H}\right]$ vortioxetine or $\left[{ }^{3} \mathrm{H}\right]$ imipramine. Cell suspensions were incubated for $2 \mathrm{~h}$ at $4{ }^{\circ} \mathrm{C}$ on a nutator, followed by exposure to a Maxima ML-3500 S UV-A light (Spectronics Corporation) in a $4^{\circ} \mathrm{C}$ cold room on ice for $15 \mathrm{~min}$. After ultraviolet light exposure, cells were pelleted $(1,500 \mathrm{~g}, 10 \mathrm{~min})$, the supernatant fraction was removed and the pellet was stored at $-20^{\circ} \mathrm{C}$ until further analysis. For quantification of crosslinked $\left[{ }^{3} \mathrm{H}\right]$ vortioxetine and $\left[{ }^{3} \mathrm{H}\right]$ imipramine, cell pellets were solubilised in $1 \%$ Triton $\mathrm{X}-100$ in $20 \mathrm{mM}$ Tris- $\mathrm{HCl}, \mathrm{pH} 7.5$, containing protease inhibitors for $1 \mathrm{~h}$ at $4{ }^{\circ} \mathrm{C}$ on a nutator. After solubilization, the lysate was centrifuged $(20,000 \mathrm{~g}, 10 \mathrm{~min})$ and the supernatant was immunopurified as described above. The purified protein was analysed by SDS-PAGE and immunoblot. The PVDF membrane was then cut between each lane to separate samples, and each lane was further cut into three segments covering 20-50, 50-100 and $>100 \mathrm{kDa}$. Each membrane segment was soaked in scintillation fluid (Ecoscint A, National diagnostics) and counted in a Tri Carb 2900 TR liquid scintillation counter (Perkin-Elmer). Non-ultraviolet-exposed samples were in all cases assayed in parallel experiments.

Data analysis. All data analyses were performed using Prism 6 software (GraphPad, Inc.). For determination of the Michaelis-Menten constant $\left(K_{\mathrm{m}}\right)$, data from $K_{\mathrm{m}}$ assays were fitted by equation (1),

$$
\% \text { specific uptake }=100 /\left(1+10^{\left(\left(\log K_{\mathrm{m}}-\log [5-H T]\right) \times \text { Hill slope }\right)}\right)
$$

For determination of $\mathrm{IC}_{50}$ values, data from $\left[{ }^{3} \mathrm{H}\right] 5-\mathrm{HT}$ uptake inhibition assays were fitted by equation (2),

$$
\% \text { specific uptake }=100 /\left(1+10^{\left(\left(\log \mathrm{IC}_{50}-\log [\text { inhibitor }]\right) \times \text { Hill slope }\right)}\right)
$$

The $K_{\mathrm{i}}$ for inhibitors were calculated from $\mathrm{IC}_{50}$ values using the Cheng-Prusoff equation (equation (3)) $)^{55}$,

$$
K_{\mathrm{i}}=\mathrm{IC}_{50} /\left(1+\left([\mathrm{L}] / K_{\mathrm{m}}\right)\right)
$$

where $[\mathrm{L}]$ is the concentration of $\left[{ }^{3} \mathrm{H}\right] 5-\mathrm{HT}$. Ki values were compared using Student's $t$-test unless otherwise indicated. For determination of $K_{\mathrm{d}}$ values, data from saturation binding assays were fitted by equation (4),

$$
\text { Specific binding }=\text { Maximum specific binding } \times[\text { ligand }] /\left(K_{\mathrm{d}}+[\text { ligand }]\right)
$$

\section{References}

1. Torres, G. E., Gainetdinov, R. R. \& Caron, M. G. Plasma membrane monoamine transporters: structure, regulation and function. Nat. Rev. Neurosci. 4, 13-25 (2003).

2. Kristensen, A. S. et al. SLC6 neurotransmitter transporters: structure, function, and regulation. Pharmacol. Rev. 63, 585-640 (2011).

3. Andersen, J., Kristensen, A. S., Bang-Andersen, B. \& Strømgaard, K. Recent advances in the understanding of the interaction of antidepressant drugs with serotonin and norepinephrine transporters. Chem. Commun. 3677-3692, doi:10.1039/b903035m (2009).
4. Yamashita, A., Singh, S. K., Kawate, T., Jin, Y. \& Gouaux, E. Crystal structure of a bacterial homologue of $\mathrm{Na}^{+} / \mathrm{Cl}^{-}$-dependent neurotransmitter transporters. Nature 437, 215-223 (2005).

5. Krishnamurthy, H. \& Gouaux, E. X-ray structures of LeuT in substrate-free outward-open and apo inward-open states. Nature 481, 469-474 (2012).

6. Malinauskaite, L. et al. A mechanism for intracellular release of $\mathrm{Na}^{+}$by neurotransmitter/sodium symporters. Nat. Struct. Mol. Biol. 21, 1006-1012 (2014).

7. Penmatsa, A., Wang, K. H. \& Gouaux, E. X-ray structure of dopamine transporter elucidates antidepressant mechanism. Nature 503, 85-90 (2013).

8. Penmatsa, A., Wang, K. H. \& Gouaux, E. X-ray structures of Drosophila dopamine transporter in complex with nisoxetine and reboxetine. Nat. Struct. Mol. Biol. 22, 506-508 (2015).

9. Wang, H. et al. Structural basis for action by diverse antidepressants on biogenic amine transporters. Nature 503, 141-145 (2013).

10. Wang, K. H., Penmatsa, A. \& Gouaux, E. Neurotransmitter and psychostimulant recognition by the dopamine transporter. Nature 521, 322-327 (2015).

11. Barker, E. L., Moore, K. R., Rakhshan, F. \& Blakely, R. D. Transmembrane domain I contributes to the permeation pathway for serotonin and ions in the serotonin transporter. J. Neurosci. 19, 4705-4717 (1999).

12. Henry, L. K. et al. Tyr-95 and Ile-172 in transmembrane segments 1 and 3 of human serotonin transporters interact to establish high affinity recognition of antidepressants. J. Biol. Chem. 281, 2012-2023 (2006).

13. Andersen, J. et al. Location of the antidepressant binding site in the serotonin transporter: importance of Ser-438 in recognition of citalopram and tricyclic antidepressants. J. Biol. Chem. 284, 10276-10284 (2009).

14. Andersen, J. et al. Mutational mapping and modeling of the binding site for (S)-citalopram in the human serotonin transporter. J. Biol. Chem. 285, 2051-2063 (2010).

15. Koldsø, H. et al. The two enantiomers of citalopram bind to the human serotonin transporter in reversed orientations. J. Am. Chem. Soc. 132, 1311-1322 (2010).

16. Sørensen, L. et al. Interaction of antidepressants with the serotonin and norepinephrine transporters: mutational studies of the S1 substrate binding pocket. J. Biol. Chem. 287, 43694-43707 (2012).

17. Zhou, Z. et al. LeuT-desipramine structure reveals how antidepressants block neurotransmitter reuptake. Science 317, 1390-1393 (2007).

18. Zhou, Z. et al. Antidepressant specificity of serotonin transporter suggested by three LeuT-SSRI structures. Nat. Struct. Mol. Biol. 16, 652-657 (2009).

19. Singh, S. K., Yamashita, A. \& Gouaux, E. Antidepressant binding site in a bacterial homologue of neurotransmitter transporters. Nature 448, 952-956 (2007).

20. Singh, S. K., Piscitelli, C. L., Yamashita, A. \& Gouaux, E. A competitive inhibitor traps LeuT in an open-to-out conformation. Science 322, 1655-1661 (2008).

21. Shi, L., Quick, M., Zhao, Y., Weinstein, H. \& Javitch, J. A. The mechanism of neurotransmitter:sodium symporter-inward release of $\mathrm{Na}^{+}$and substrate is triggered by substrate in a second binding site. Mol. Cell 30, 667-677 (2008).

22. Plenge, P. et al. Steric hindrance mutagenesis in the conserved extracellular vestibule impedes allosteric binding of antidepressants to the serotonin transporter. J. Biol. Chem. 287, 39316-39326 (2012).

23. Wang, L., Xie, J. \& Schultz, P. G. Expanding the genetic code. Annu. Rev. Biophys. Biomol. Struct. 35, 225-249 (2006).

24. Liu, C. C. \& Schultz, P. G. Adding new chemistries to the genetic code. Annu. Rev. Biochem. 79, 413-444 (2010).

25. Davis, L. \& Chin, J. W. Designer proteins: applications of genetic code expansion in cell biology. Nat. Rev. Mol. Cell Biol. 13, 168-182 (2012).

26. Wang, L., Brock, A., Herberich, B. \& Schultz, P. G. Expanding the genetic code of Escherichia coli. Science 292, 498-500 (2001).

27. Mori, H. \& Ito, K. Different modes of SecY-SecA interactions revealed by site-directed in vivo photo-cross-linking. Proc. Natl Acad. Sci. USA 103, 16159-16164 (2006).

28. Hino, N. et al. Protein photo-cross-linking in mammalian cells by site-specific incorporation of a photoreactive amino acid. Nat. Methods 2, 201-206 (2005)

29. Chin, J. W., Martin, A. B., King, D. S., Wang, L. \& Schultz, P. G. Addition of a photocrosslinking amino acid to the genetic code of Escherichia coli. Proc. Natl Acad. Sci. USA 99, 11020-11024 (2002).

30. Takimoto, J. K., Adams, K. L., Xiang, Z. \& Wang, L. Improving orthogonal tRNA-synthetase recognition for efficient unnatural amino acid incorporation and application in mammalian cells. Mol. Biosyst. 5, 931-934 (2009).

31. Grunbeck, A. et al. Genetically encoded photo-cross-linkers map the binding site of an allosteric drug on a G protein-coupled receptor. ACS Chem. Biol. 7, 967-972 (2012).

32. Grunbeck, A., Huber, T., Sachdev, P. \& Sakmar, T. P. Mapping the ligand-binding site on a $\mathrm{G}$ protein-coupled receptor (GPCR) using genetically encoded photocrosslinkers. Biochemistry 50, 3411-3413 (2011). 
33. Valentin-Hansen, L. et al. Mapping substance P binding sites on the neurokinin-1 receptor using genetic incorporation of a photoreactive amino Acid. J. Biol. Chem. 289, 18045-18054 (2014).

34. Coin, I., Perrin, M. H., Vale, W. W. \& Wang, L. Photo-cross-linkers incorporated into G-protein-coupled receptors in mammalian cells: a ligand comparison. Angew. Chem. Int. Ed. 50, 8077-8081 (2011).

35. Ye, S. et al. Site-specific incorporation of keto amino acids into functional G protein-coupled receptors using unnatural amino acid mutagenesis. J. Biol. Chem. 283, 1525-1533 (2008).

36. Ye, S., Huber, T., Vogel, R. \& Sakmar, T. P. FTIR analysis of GPCR activation using azido probes. Nat. Chem. Biol. 5, 397-399 (2009).

37. Ye, S. et al. Tracking G-protein-coupled receptor activation using genetically encoded infrared probes. Nature 464, 1386-1389 (2010).

38. Coin, I. et al. Genetically encoded chemical probes in cells reveal the binding path of urocortin-I to CRF class B GPCR. Cell 155, 1258-1269 (2013).

39. Klippenstein, V., Ghisi, V., Wietstruk, M. \& Plested, A. J. Photoinactivation of glutamate receptors by genetically encoded unnatural amino acids. J. Neurosci. 34, 980-991 (2014).

40. Zhu, S. et al. Genetically encoding a light switch in an ionotropic glutamate receptor reveals subunit-specific interfaces. Proc. Natl Acad. Sci. USA 111, 6081-6086 (2014).

41. Ye, S., Riou, M., Carvalho, S. \& Paoletti, P. Expanding the genetic code in Xenopus laevis oocytes. Chembiochem. 14, 230-235 (2013).

42. Andersen, J. et al. Binding of the multimodal antidepressant drug vortioxetine to the human serotonin transporter. ACS Chem. Neurosci. 6, 1892-1900 (2015).

43. Koban, F. et al. A salt bridge linking the first intracellular loop with the $\mathrm{C}$ terminus facilitates the folding of the serotonin transporter. J. Biol. Chem. 290, 13263-13278 (2015)

44. Mørk, A. et al. Pharmacological effects of Lu AA21004: a novel multimodal compound for the treatment of major depressive disorder. J. Pharmacol. Exp. Ther. 340, 666-675 (2012).

45. Bang-Andersen, B. et al. Discovery of 1-[2-(2,4-dimethylphenylsulfanyl)phenyl] piperazine (Lu AA21004): a novel multimodal compound for the treatment of major depressive disorder. J. Med. Chem. 54, 3206-3221 (2011).

46. Talvenheimo, J., Fishkes, H., Nelson, P. J. \& Rudnick, G. The serotonin transporter-imipramine "receptor". J. Biol. Chem. 258, 6115-6119 (1983).

47. Kumar, V. et al. Novel azido-iodo photoaffinity ligands for the human serotonin transporter based on the selective serotonin reuptake inhibitor (S)-citalopram. J. Med. Chem. 58, 5609-5619 (2015).

48. Dahal, R. A. et al. Computational and biochemical docking of the irreversible cocaine analog RTI 82 directly demonstrates ligand positioning in the dopamine transporter central substrate-binding site. J. Biol. Chem. 289, 29712-29727 (2014).

49. Parnas, M. L. et al. Labeling of dopamine transporter transmembrane domain 1 with the tropane ligand $N$-[4-(4-azido-3-[ $\left.{ }^{125} \mathrm{I}\right]$ iodophenyl)butyl $]-2 \beta$ carbomethoxy-3 $\beta$-(4-chlorophenyl) tropane implicates proximity of cocaine and substrate active sites. Mol. Pharmacol. 73, 1141-1150 (2008).

50. Vaughan, R. A., Agoston, G. E., Lever, J. R. \& Newman, A. H. Differential binding of tropane-based photoaffinity ligands on the dopamine transporter. J. Neurosci. 19, 630-636 (1999).
51. Sinning, S. et al. Binding and orientation of tricyclic antidepressants within the central substrate site of the human serotonin transporter. J. Biol. Chem. 285, 8363-8374 (2010).

52. Sarker, S. et al. The high-affinity binding site for tricyclic antidepressants resides in the outer vestibule of the serotonin transporter. Mol. Pharmacol. 78, 1026-1035 (2010).

53. Andersen, J. et al. Molecular basis for selective serotonin reuptake inhibition by the antidepressant agent fluoxetine (Prozac). Mol. Pharmacol. 85, 703-714 (2014).

54. Huber, T. \& Sakmar, T. P. Chemical biology methods for investigating G protein-coupled receptor signaling. Chem. Biol. 21, 1224-1237 (2014).

55. Cheng, Y. \& Prusoff, W. H. Relationship between the inhibition constant $\left(\mathrm{K}_{\mathrm{I}}\right)$ and the concentration of inhibitor which causes 50 per cent inhibition $\left(\mathrm{I}_{50}\right)$ of an enzymatic reaction. Biochem. Pharmacol. 22, 3099-3108 (1973).

\section{Acknowledgements}

We are grateful for the financial support from the Lundbeck Foundation, Aase og Ejnar Danielsens Fond, Brødrene Hartmanns Fond, Torben og Alice Frimodts Fond, Augustinusfonden, Fonden til Lægevidenskabens Fremme and Læge Sofus Carl Emil Friis og Hustru Olga Friis' legat. We thank Dr Birgit Schiøtt for providing the imipramine docking model. Silvia Inderbinen is thanked for help in generation and initial characterization of the hSERT mutants. We thank Drs Stephan A. Pless and Claus J. Loland for the critical comments on the manuscript.

\section{Author contributions}

H.R., J.A., T.H., T.P.S. and K.S. designed the research. H.R., J.A., L.S. and M.P. performed the experiments, and analysed the results together with T.P.S. and K.S. B.B.-A. contributed new analytical tools. J.A., T.P.S. and K.S. supervised the research. H.R., J.A., L.S. and K.S. wrote the manuscript with help from T.P.S.

\section{Additional information}

Supplementary Information accompanies this paper at http://www.nature.com/ naturecommunications

Competing financial interests: B.B.-A. is an employee of H. Lundbeck A/S. The other authors declare no competing financial interests.

Reprints and permission information is available online at http://npg.nature.com/ reprintsandpermissions/

How to cite this article: Rannversson, H. et al. Genetically encoded photocrosslinkers locate the high-affinity binding site of antidepressant drugs in the human serotonin transporter. Nat. Commun. 7:11261 doi: 10.1038/ncomms11261 (2016).

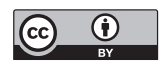

This work is licensed under a Creative Commons Attribution 4.0 International License. The images or other third party material in this article are included in the article's Creative Commons license, unless indicated otherwise in the credit line; if the material is not included under the Creative Commons license, users will need to obtain permission from the license holder to reproduce the material. To view a copy of this license, visit http://creativecommons.org/licenses/by/4.0/ 